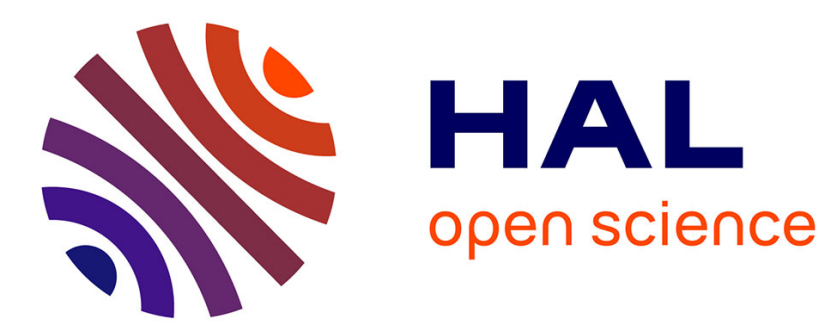

\title{
Fast reconstruction of image deformation field using radial basis function
}

\author{
Lukáš Ručka, Igor Peterlík
}

\section{To cite this version:}

Lukáš Ručka, Igor Peterlík. Fast reconstruction of image deformation field using radial basis function. ISBI2017 - International Symposium on Biomedical Imaging, Apr 2017, Melbourne, Australia. hal01445483

\section{HAL Id: hal-01445483 \\ https://inria.hal.science/hal-01445483}

Submitted on 24 Jan 2017

HAL is a multi-disciplinary open access archive for the deposit and dissemination of scientific research documents, whether they are published or not. The documents may come from teaching and research institutions in France or abroad, or from public or private research centers.
L'archive ouverte pluridisciplinaire HAL, est destinée au dépôt et à la diffusion de documents scientifiques de niveau recherche, publiés ou non, émanant des établissements d'enseignement et de recherche français ou étrangers, des laboratoires publics ou privés. 


\title{
FAST RECONSTRUCTION OF IMAGE DEFORMATION FIELD USING RADIAL BASIS FUNCTION
}

\author{
Lukáš Ručka $\quad$ Igor Peterlík $k^{\ddagger}$ \\ $\ddagger$ Faculty of Informatics, Masaryk University, Czech Republic \\ $\dagger$ Institute of Computer Science, Masaryk University, Czech Republic \\ * Inria, France
}

\begin{abstract}
Fast and accurate registration of image data is a key component of computer-aided medical image analysis. Instead of performing the registration directly on the input images, many algorithms compute the transformation using a sparse representation extracted from the original data. However, in order to apply the resulting transformation onto the original images, a dense deformation field has to be reconstructed using a suitable inter-/extra-polation technique.

In this paper, we employ the radial basis function (RBF) to reconstruct the dense deformation field from a sparse transformation computed by a model-based registration. Various kernels are tested using different scenario. The dense deformation field is used to warp the source image and compare it quantitatively to the target image using two different metrics. Moreover, the influence of the number and distribution of the control points required by the RBF is studied via two different scenarios. Beside the accuracy, the performance of the method accelerated using a GPU is reported.
\end{abstract}

Index Terms - image warping, image interpolation, medical imaging, non-rigid registration

\section{INTRODUCTION}

Image registration is the process of overlaying multiple images of the same scene, acquired by different modalities, from various viewpoints and/or at different times [1].

The goal of registration is thus to find the optimal transformation $W$, mapping coordinates from source image $S$ onto target image $T$ [2]. From mathematical perspective, image registration an is optimization of objective function given as

$$
M(W(S), T)+R_{M}(W)
$$

which consists of similarity measure $M$, typically complemented with a regularization term $R_{M}$, which introduces $a$ priori knowledge into the optimization process. It is often used to limit the set of possible transformations to those which are physically feasible [3].

Instead of using the dense representation typically represented by the images $T$ and $S$, the registration process can be designed as optimization of objective function evaluated on a sparse representations $t$ and $s$, typically geometric primitives such as points or convexes, extracted from the original data. In this case, a sparse transformation $w$ is computed and the metric $M$ is usually replaced by a different criterion $m$ such as Euclidean or Hausdorff distance. The sparse registration is then summarized as follows:

SR1 Sparse representation $s$ and $t$ such as set of features or a mesh is extracted from the source and target images.

SR2 Sparse transformation $w$ is computed by optimization of the criteria $m$ applied to $t$ and $w(s)$. The process can be again regularized by a priori information introduced via a suitable term.

SR3 Dense transformation $W$ is constructed from $w$ via suitable interpolation or approximation technique [4].

SR4 Similarity measure $M$ is evaluated over target image $T$ and transformed source image $W(S)$.

The approach described above has been employed in a large number of algorithms. A finite element model (FE model) is core component of ITK filters for physics-based non-rigid registration presented in $[5,6]$. They use the data from image block matching as input for a finite element modeling of the underlying tissue, which yields sparse transformation. The deformation field $W$ is then obtained as a result of hybrid iterative approximation-interpolation algorithm with outlier block rejection step. Non-linear finite element modeling has been also deployed for brain registration [7], interpolating the sparse transformation with algorithm proposed in [8]. The interpolation is based on B-spline deformation of Gaussian curvature. A compound dense transformation is used in [9], consisting of free-form deformation with homogeneous coarse grid and B-spline convolution kernel for interpolation inbetween the latices. This approach has been adapted in [4], improving the results with a cross-cumulative residual entropy metric. The dense deformation field, obtained by the CLI-TPS algorithm [10] is subjected to constraint-based smoothing in lung CT registration [11]. The constraints presented ensure that the dense field preserves the topological properties of the original structure. 
The sparse transformation obtained by the registration process often provides values in positions which are not organized in a regular structure. Therefore, an interpolation from scatter data such as radial basis functions (RBF) interpolation is used to compute the dense transformation [12]. A series of criteria for sparse deformation field interpolation via RBF was given in [13]. Furthermore, computational efficiency of RBF interpolation has been addressed in [14] and later evaluated [15]. Remarkably, in this work, the uncertainty at input was greater than the error introduced by approximation of RBF solution.

In this paper, we focus mainly on steps SR3 and SR4 as given in the list above. Both SR1 and SR2 are performed by an external registration process. We have chosen a meshbased registration presented in [16]. The method presented therein is regularized with co-rotational formulation of linear elasticity, therefore the resulting sparse elastic transformation is physically admissible and capable of handling very large displacements. We apply the RBF to convert the sparse transformation and study the influence of the RBF method for both the domain covered by the FE mesh (interpolating mode), as well as in the areas outside of the mesh (RBF in extrapolating mode). Method is assessed using two different image metrics and visual inspection for different RBF splines, resolutions of the image data and different inputs provided by the sparse registration.

\section{METHOD}

In this section, we first shortly describe the RBF interpolation method. Then, we couple this technique with a sparse FEbased registration in order to obtain dense transformation that can be used to warp 3D images.

\subsection{Radial Basis Function Interpolation}

In general, the RBF interpolation in point $x$ is given as a weighted sum of radial base function $\varphi(r)$, centered in control points $X_{i}$ :

$$
f(\mathbf{x})=A \cdot \mathbf{x}+\sum_{i} w_{i} \cdot \varphi\left(\left\|X_{i}-\mathbf{x}\right\|\right)
$$

where $A$ is an affine transformation term. The interpolation is computed in two steps. First, given known values of the function $f$ in $m$ control points $X_{j}, j=1 \ldots m$, the vector w of weights is found by substituting $f\left(X_{j}\right)$ for $f(x)$ on the left-hand side, and $X_{j}$ for $x$ on the right-hand side of the Eq. 2 and solving the resulting system of linear equations. Second, for any new position $x$, the value $f(x)$ is computed directly by Eq. 2. By its nature, RBF interpolation is unidimensional; interpolation of multidimensional values is considered as combination of independent unidimensional transformations. As for the choice of the base function, in this paper, we consider following options: $\varphi(r)=r^{2} \cdot \log (r)$ Thin plate splines (TPS) - solution of bending energy functional, minimizing second order bending energy potential in 2D [15].

$\varphi(r)=r^{2}$ (biharmonic RBF spline) - solution of bending energy functional, minimizing second order bending energy potential in 3D [15].

$\varphi(r)=r^{3}$ (triharmonic RBF spline) - solution of bending energy functional, minimizing third order bending energy potential in 3D [15].

$\varphi(r)=r^{3} \cdot \log (r), \varphi(r)=r^{2} \cdot \log (\log (r)), \varphi(r)=r \cdot \log$ $(\log (r))$ - test base functions, illustrating different aspects of dense transformations they yield ${ }^{1}$. In the following, these base functions are referred to as excited triharmonic (ETRI), damped thin-plate spline (DTPS) and excited linear with damping (EDL) respectively.

\subsection{From Sparse Registration to Dense Transformation}

In the scenario considered in this paper, we employ the surfacematching registration presented in [16] which performs a meshto-mesh registration based on constrained biomechanical simulation. Indeed, the method does not work directly with the images, but it is necessary to extract the sparse representation given by the FE meshes of the object of interest in source and target configurations, $\mathcal{M}_{s}$ and $\mathcal{M}_{t}$, respectively. During the registration process regularized by the co-rotational formulation of the linear elasticity, the $\mathcal{M}_{s}$ is deformed due to the application of sliding constraints which are used to match the shape of $\mathcal{M}_{s}$ to the shape of $\mathcal{M}_{t}$. The procedure results in the registered mesh $\tilde{\mathcal{M}}_{s}$ : although being deformed, this mesh has the same topological structure as $\mathcal{M}_{s}$. The vectors of nodal positions of the two meshes constitute the sparse transformation computed by the mesh-to-mesh registration.

Now, to obtain the dense transformation, RBF is employed as follows. Using the nomenclature introduced in the previous section, the nodes of the deformed mesh $\tilde{\mathcal{M}}_{s}$ are used as control points and $f\left(\tilde{\mathbf{n}}_{s}\right)=\mathbf{n}_{s}-\tilde{\mathbf{n}}_{s}$ is the $3 \mathrm{D}$ function which is used to compute three weight vectors $\mathbf{w}_{x}, \mathbf{w}_{y}, \mathbf{w}_{z}$ and affine transformation $\mathbf{A}$. Then, the value of transformation is interpolated over the original image, i.e., the Eq. 2 is evaluated in each voxel of the image.

\section{RESULTS}

\subsection{Data and Sparse Registration}

The evaluation was performed used a set of CT scans of a female pig liver (target organ) and kidneys (auxiliary organs). Two abdominal CT scans were acquired in flank and supine positions with SOMATOM ${ }^{\circledR}$ Definition AS 128 device. A bounding box containing the liver was extracted from the original

\footnotetext{
${ }^{1}$ As noted in [12], certain base functions can yield good results even though they are not mathematically justified.
} 


\begin{tabular}{|c|c|c|c|c|c|c|c|c|c|c|c|c|}
\hline \multirow{3}{*}{ Interpolation } & \multicolumn{4}{|c|}{ Liver only } & \multicolumn{4}{|c|}{ Liver + kidneys } & \multicolumn{4}{|c|}{ Cross-scenario improvement } \\
\hline & \multicolumn{2}{|c|}{ Dice } & \multicolumn{2}{|c|}{ MMI } & \multicolumn{2}{|c|}{ Dice } & \multicolumn{2}{|c|}{ MMI } & \multicolumn{2}{|c|}{ Dice } & \multicolumn{2}{|c|}{ MMI } \\
\hline & LR & HR & LR & HR & LR & HR & LR & HR & LR & HR & LR & HR \\
\hline No interpolation & 0.5123 & 0.5148 & -0.1032 & -0.098 & 0.5123 & 0.5148 & -0.1032 & -0.098 & - & - & - & - \\
\hline EDL & 0.9333 & 0.9495 & $-0.2395^{\star}$ & -0.2237 & 0.9333 & 0.9496 & -0.2586 & -0.2457 & 0 & 0.0001 & -0.0191 & -0.0219 \\
\hline Biharmonic & 0.9341 & 0.9519 & -0.2306 & $-0.2301^{\star}$ & 0.9339 & 0.9519 & $-0.2635^{\star}$ & $-0.2607^{\star}$ & -0.0002 & 0 & $-0.0329^{\dagger}$ & $-0.0306^{\dagger}$ \\
\hline DTPS & 0.9358 & 0.9526 & $-0.239^{\dagger}$ & $-0.2247^{\dagger}$ & $0.9357^{\dagger}$ & $0.9526^{\star}$ & $-0.2608^{\dagger}$ & $-0.2543^{\dagger}$ & -0.0001 & 0 & -0.0218 & -0.0296 \\
\hline Thinplate & $0.9363^{\dagger}$ & $0.9528^{\dagger}$ & -0.2236 & -0.2187 & $0.936^{\star}$ & $0.9526^{\dagger}$ & -0.2533 & -0.2435 & -0.0003 & -0.0002 & -0.0297 & -0.0247 \\
\hline Triharmonic & $0.9371^{\star}$ & $0.9531^{\star}$ & -0.179 & -0.171 & 0.8531 & 0.8621 & -0.2495 & -0.2335 & -0.084 & -0.091 & $-0.0705^{\star}$ & $-0.0625^{\star}$ \\
\hline ETRI & 0.9286 & 0.9446 & -0.1703 & -0.1622 & 0.1453 & 0.1528 & -0.0547 & -0.0377 & -0.7833 & -0.7918 & 0.1156 & 0.1245 \\
\hline
\end{tabular}

Table 1: Evaluation of target organ driven warping with respect to Dice and Mattes MI metrics. Blue ${ }^{\star}$ and green ${ }^{\dagger}$ colors denote the best and the second best result achieved for given metric (maximum for Dice and minimum for Mattes MI).

scans in two versions: first, the original resolution was preserved resulting in high-resolution images (HR) composed of $480 \times 452 \times 388$ voxels with spacing $0.5 \times 0.5 \times 0.5 \mathrm{~mm}^{3}$. The same volume was resampled to lower resolution (LR): $240 \times 226 \times 194$ voxels, spacing $1 \times 1 \times 1 \mathrm{~mm}^{3}$.

The FE meshes extracted from the original images were composed of 843 nodes (liver) and 165 and 207 nodes for kidneys. The sparse registration was performed using the method presented in [16]: the supine configurations of the liver and kidney meshes were registered to the corresponding flank configurations; the registration of each organ was performed separately resulting in three complementary sparse transformations. While the kidneys displayed important translation and rotation, the liver underwent large deformations resulting in large displacements of lateral lobes (up to $6 \mathrm{~cm}$ ).

\subsection{Testing Scenarios}

The dense transformation was constructed for each base function from section 2.1. Further, two different scenarios were considered. Since we are interested in registration of liver and its surroundings, the dense transformation was first constructed using only the control points provided by the liver mesh. In the second scenario, the control points obtained by the registration of kidneys as auxiliary organs were also added to the liver mesh nodes to augment the set of control nodes. Since the three organs perform relatively independent movements, the goal was to test the capabilities of the RBF method.

All the scenarios were evaluated for both the low- and highresolution images using two metrics: the interpolation mode was evaluated by the Dice metric [17] computed using the binary maps of the liver. Further, Mattes mutual information (MMI) [18] was computed using the target image and the source image warped by the dense transformation. Hence, the Dice metric was used to assess the interpolating mode locally, while MMI was employed as a global metric quantifying the quality of warping that was obtained by combining the physicsbased approach (the sparse registration) and geometric RBF method.

\subsection{Evaluation Using Warped Images}

Dense transformations were constructed using the RBF method using a PC equipped with Intel ${ }^{\circledR}$ Core $^{\mathrm{TM}}$ i7-3770 CPU running $3.40 \mathrm{GHz}, 32 \mathrm{~GB}$ RAM and NVIDIA GeForce GTX 560. The second phase of the method, where the RBF is evaluated for each voxel, was accelerated using the GPU. The computing times are reported in Tab. 2.

\begin{tabular}{|l|c|c|c|c|c|c|}
\hline \multirow{2}{*}{ Scenario } & \multicolumn{2}{|c|}{ GPU L [s] } & \multicolumn{2}{c|}{ GPU L+K [s] } & \multicolumn{2}{c|}{ CPU L [s] } \\
\cline { 2 - 7 } & LR & HR & LR & HR & LR & HR \\
\hline \hline EDL & 2.64 & 21.13 & 4.76 & 37.88 & 338.9 & 2695 \\
\hline Biharmonic & 1.70 & 13.45 & 3.23 & 25.64 & 132.2 & 1052 \\
\hline DTPS & 2.59 & 20.57 & 4.69 & 38.17 & 333.8 & 2645 \\
\hline Thinplate & 2.19 & 17.39 & 4.10 & 32.61 & 231.1 & 1833 \\
\hline Triharmonic & 1.72 & 13.62 & 3.24 & 25.79 & 133.6 & 1067 \\
\hline ETRI & 2.17 & 17.20 & 4.06 & 34.22 & 253.2 & 2012 \\
\hline
\end{tabular}

Table 2: Processing times of individual scenarios - liver (L), liver + kidneys $(\mathrm{L}+\mathrm{K})-$ retrieved via code instrumentation. $\mathrm{CPU}$ times for liver registration (CPU L) are provided for illustration.

The quantitative results showing the MMI and Dice metrics are reported in Tab. 1. Beside the values of metrics, the relative differences are shown on the right-hand side. The best results are displayed in colors. Moreover, an illustration of results is shown in Fig. 1. We have deliberately chosen an axial slice located below the liver, which represents the area covered with the control points, in order to illustrate the behavior of the extrapolated dense deformation field for different choices of RBF spline.

Both TPS and triharmonic splines perform well in terms of Dice metric. Nonetheless, although the liver keeps its shape, the near vicinity suffers from vast drifts. While triharmonic spline renders the segmented liver smoother, TPS creates artificial concavities. Furthermore, the results of EDL, biharmonic and DTPS splines display visual similarity, without any noticeable issues. However, none of the splines allows for approaching the deformation of the flank image outside of the liver region. 


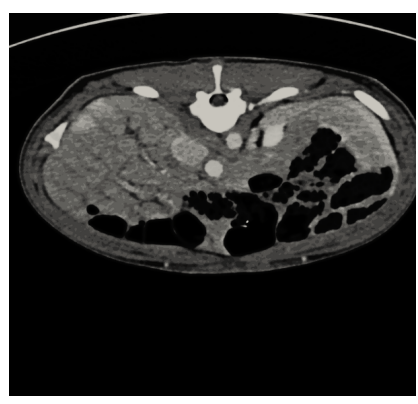

(a)

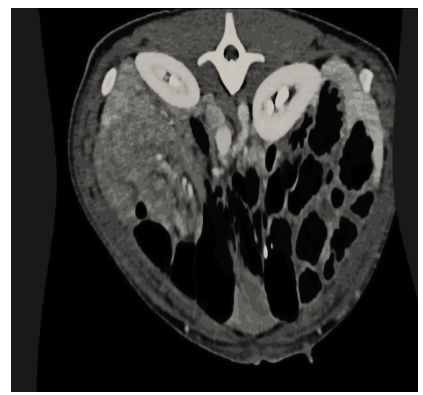

(c)

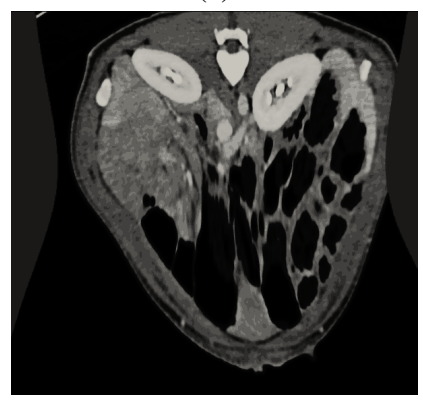

(e)

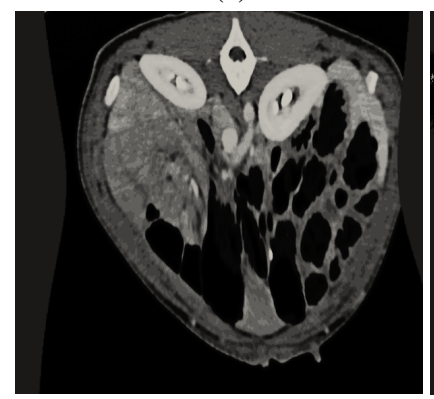

(g)

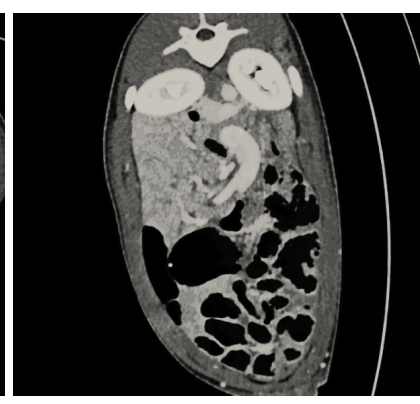

(b)

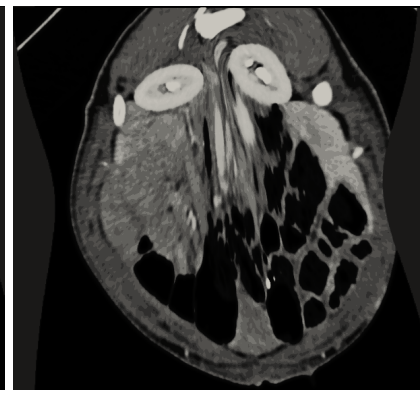

(d)

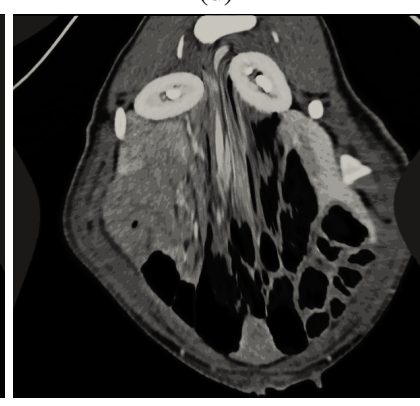

(f)

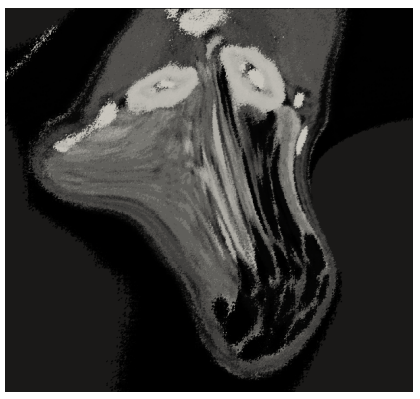

(h)
Fig. 1: Comparison of the warped images obtained via different RBF splines.

(a): source image, (b): target image

On the left, scenarios using control points only from liver mesh. On the right, scenarios using control points from liver and kidney meshes. Types of the RBF spline:

(c),(d): biharmonic,

(e),(f): DTPS,

(g): EDL, (h): triharmonic.
In the scenario using also the registered kidneys as auxiliary sources of control points, the Dice metric performs best with both Thin plate splines and DTPS. However, TPS achieves inferior score in terms of Mattes MI metric. On the other hand, the performance of other spline is acceptable for Mattes mutual information (MMI) metric as well. The best results for MMI are achieved using the biharmonic spline. Upon visual inspection of the segmented liver, all three results look naturally. Nevertheless, the TPS seems to emphasize bulges on liver surface. Furthermore, visual inspection shows slight image deterioration along the borders, clearly visible for TPS warp. In addition, the DTPS exhibits stable results throughout distinct scenarios.

Out of all RBF splines tested, the ETRI performs the worst in terms of both Dice and MMI metrics. Upon visual inspection, the surface of the liver seems to suffer from material drift. Inspection of the deformation field reveals large displacements in image parts uncovered by RBF control points, rendering the intensity image completely useless. Correspondingly, adding displacement contribution from kidneys lead to complete deformation field breakdown, thus degenerating segmented liver to point cloud.

\section{DISCUSSION AND CONCLUSIONS}

While including auxiliary organs slightly corrupts the Dice metric, it improves the MMI metric in all cases where single organ warping does not already exhibit deterioration. The improvement is, however, only decimal order higher than metric sensitivity, computed as the difference of MMI values for noninterpolated warps. Thus, as the changes in Dice metric are negligible $(<0.0003)$, including auxiliary organs improves the overall MMI result. However, neither the improvement alone, nor MMI score guarantee that the result is acceptable. For example, the triharmonic spline achieves reasonably-high score in MMI, yet the image is severely degenerated. The only significant deterioration of Dice result in combination with improvement in MMI suggest degenerated image.

It is equally important to understand the nature of all the splines considered in this paper: the further the inter-/extrapolated voxel from a control point, the stronger the effect of given control point. The data used also displays strong rotational changes for kidneys, as pig lacks ligaments which are present in human body. This observations combined probably explain the poor performance of the triharmonic and higher splines. As noted in [13], the distance property can introduce significant artifacts.

Beside testing other intensity-based metrics, we plan to compare this warping technique to other global interpolation methods such as natural neighbor interpolation. However, exploring different interpolation frameworks or base functions with better locality properties (e.g. Gaussian) is beyond the scope of this paper and will be subject of the future work. 


\section{REFERENCES}

[1] B. Zitová and J. Flusser, "Image Registration Methods: A Survey," Image and Vision Computing, vol. 21, no. 11, pp. 977-1000, 2003.

[2] C. A. Glasbey and K. V. Mardia, "A review of imagewarping methods," J. Appl. Stat., vol. 25, no. 2, pp. 155-171, 1998.

[3] A. Sotiras, C. Davatzikos, and N. Paragios, "Deformable Medical Image Registration: A Survey," IEEE Transactions on Medical Imaging, vol. 32, no. 7, pp. 1153-1190, jul 2013.

[4] F. Wang and B. C. Vemuri, "Non-Rigid Multi-Modal Image Registration Using Cross-Cumulative Residual Entropy," International Journal of Computer Vision, vol. 74, no. 2, pp. 201-215, 2007.

[5] O. Clatz, H. Delingette, I. F. Talos, A. J. Golby, Ron Kikinis, Ferenc A. Jolesz, N. Ayache, and S. K. Warfield, "Robust Nonrigid Registration to Capture Brain Shift from Intraoperative MRI,' IEEE Transactions on Medical Imaging, vol. 24, no. 11, pp. 1417-1427, 2005.

[6] Y. Liu, A. Kot, F. Drakopoulos, C. Yao, A. Fedorov, A. Enquobahrie, O. Clatz, and N. Chrisochoides, "An ITK Implementation of a Physics-Based Non-Rigid Registration Method for Brain Deformation in Image-Guided Neurosurgery.," Frontiers in neuroinformatics, vol. 8, no. April, pp. 33, 2014.

[7] A. Mostayed, R. R. Garlapati, G. R. Joldes, A. Wittek, A. Roy, R. Kikinis, S. K. Warfield, and K. Miller, "Biomechanical Model as a Registration Tool for ImageGuided Neurosurgery: Evaluation Against BSpline Registration," Annals of Biomedical Engineering, vol. 41, no. 11, pp. 2409-2425, 2013.

[8] G. R. Joldes, A. Wittek, and K. Miller, "Cortical Surface Motion Estimation for Brain Shift Prediction," in Computational Biomechanics for Medicine, pp. 53-62. Springer, 2010.

[9] D. Mattes, D. R. Haynor, H. Vesselle, T. K. Lewellen, and W. Eubank, "PET-CT Image Registration in the Chest Using Free-Form Deformations," IEEE Transactions on Medical Imaging, vol. 22, no. 1, pp. 120-128, 2003.

[10] H. J. Johnson and G. E. Christensen, "Consistent Landmark and Intensity-Based Image Registration," IEEE Transactions on Medical Imaging, vol. 21, no. 5, pp. 450-461, 2002.

[11] B. Li, G. E. Christensen, E. a Hoffman, G. McLennan, and J. M. Reinhardt, "Pulmonary CT Image Registration and Warping for Tracking Tissue Deformation During the Respiratory Cycle Through 3D Consistent Image Registration.," Medical Physics, vol. 35, no. 12, pp. 5575-5583, 2008.

[12] I. Amidror, "Scattered Data Interpolation Methods for Electronic Imaging Systems: A Survey," Journal of Electronic Imaging, vol. 11, no. 2, pp. 157, 2002.

[13] N. Arad and D. Reisfeld, "Image Warping Using Few Anchor Points and Radial Functions," Computer Graphics Forum, vol. 14, no. 1, pp. 35-46, 1995.

[14] O. E. Livne and G. B. Wright, "Fast Multilevel Evaluation of Smooth Radial Basis Function Expansions," Electronic Transactions on Numerical Analysis, vol. 23, pp. 263-287, 2006.

[15] R. J. Lapeer, S. K. Shah, and R. S. Rowland, "An Optimised Radial Basis Function Algorithm for Fast NonRigid Registration of Medical Images," Computers in Biology and Medicine, vol. 40, no. 1, pp. 1-7, 2010.

[16] I. Peterlik, H. Courtecuisse, C. Duriez, and S. Cotin, "Model-Based Identification of Anatomical Boundary Conditions in Living Tissues," Lecture Notes in Computer Science (including subseries Lecture Notes in Artificial Intelligence and Lecture Notes in Bioinformatics), vol. 8498 LNCS, pp. 196-205, 2014.

[17] A. P. Zijdenbos, B. M. Dawant, R. A. Margolin, and A. C. Palmer, "Morphometric Analysis of White Matter Lesions in MR Images: Method and Validation," IEEE Transactions on Medical Imaging, vol. 13, no. 4, pp. 716-724, 1994.

[18] D. Mattes, D. R. Haynor, H. Vesselle, T. K. Lewellen, and W. Eubank, "Nonrigid multimodality image registration," in Medical Imaging 2001. International Society for Optics and Photonics, 2001, pp. 1609-1620. 